\title{
Approximate vortex solution of Faddeev model
}

\author{
Chang-Guang Shi \\ Department of Mathematics and Physics, \\ Shanghai University of Electric power \\ Shanghai,200090 China \\ shichangguang@shiep.edu.cn \\ Minoru Hirayama \\ Department of Mathematics and Physics, \\ Shanghai University of Electric power \\ Shanghai,200090 China \\ Department of Physics, University of Toyama, Gofuku 3190 \\ Toyama, 930-8555, Japan \\ hirayama@jodo.sci.u-toyama.ac.jp
}

\begin{abstract}
Through an Ansatz specifying the azimuthal-angle dependence of the solution, the static field equation for vortex of the Faddeev model is converted to an algebraic ordinary differential equation. An approximate analytic expression of the vortex solution is explored so that the energy per unit vortex length becomes as small as possible. It is observed that the minimum energy of vortex is approximately proportional to the integer which specifies the solution.
\end{abstract}

PACS: 11.10.Lm,02.30,Ik,03.50-z

\section{Introduction}

Faddeev model 1 was originally proposed as a model which might give rise to 3 -dimensional soliton solutions. Later it was discussed that the model might be an effective field theory describing the low energy behavior of the $S U(2)$ gauge field [2]. It can be regarded as a restricted version of the Skyrme model[3] which may be an effective field theory for hadron dynamics. Because of the high nonlinearity of Faddeev and Skyrme models, the analytic structures of solutions of these models have not yet been clarified. The numerical solutions of these models, however, exhibit quite interesting soliton properties [4]- [6]: soliton solutions of the Skyrme model have tetrahedral structures while those of the Faddeev model have knot structures [4, [5].

The Faddeev model is a model concerning the real scalar fields

$$
\boldsymbol{n}(x)=\left(n^{1}(x), n^{2}(x), n^{3}(x)\right)
$$


satisfying

$$
\boldsymbol{n}^{2}(x)=\boldsymbol{n}(x) \cdot \boldsymbol{n}(x)=\sum_{a=1}^{3} n^{a}(x) n^{a}(x)=1 .
$$

It is defined by the Lagrangian density

$$
\begin{aligned}
\mathcal{L}_{F}(x) & =c_{2} l_{2}(x)+c_{4} l_{4}(x), \\
l_{2}(x) & =\partial_{\mu} \boldsymbol{n}(x) \cdot \partial^{\mu} \boldsymbol{n}(x), \\
l_{4}(x) & =-H_{\mu \nu}(x) H^{\mu \nu}(x), \\
H_{\mu \nu}(x) & =\boldsymbol{n}(x) \cdot\left[\partial_{\mu} \boldsymbol{n}(x) \times \partial_{\nu} \boldsymbol{n}(x)\right] \\
& =\epsilon_{a b c} n^{a}(x) \partial_{\mu} n^{b}(x) \partial_{\nu} n^{c}(x),
\end{aligned}
$$

where $c_{2}$ and $c_{4}$ are constants. The static energy functional $E_{F}[\boldsymbol{n}]$ associated with $\mathcal{L}_{F}(x)$ is given by

$$
\begin{aligned}
E_{F}[\boldsymbol{n}] & =\int d V \epsilon(\boldsymbol{x}), \\
\epsilon(\boldsymbol{x}) & =c_{2} \epsilon_{2}(\boldsymbol{x})+c_{4} \epsilon_{4}(\boldsymbol{x}), \\
\epsilon_{2}(\boldsymbol{x}) & =\sum_{a=1}^{3} \sum_{i=1}^{3}\left[\partial_{i} n^{a}(\boldsymbol{x})\right]^{2}, \\
\epsilon_{4}(\boldsymbol{x}) & =\sum_{i, j=1}^{3}\left[H_{i j}(\boldsymbol{x})\right]^{2},
\end{aligned}
$$

with $\boldsymbol{x}=\left(x_{1}, x_{2}, x_{3}\right)$ and $d V=d x_{1} d x_{2} d x_{3}$. By the stereo-graphic projection, the field $\boldsymbol{n}$ can be expressed by a complex function $u$ as

$$
\boldsymbol{n}=\left(\frac{u+u^{*}}{|u|^{2}+1}, \frac{-i\left(u-u^{*}\right)}{|u|^{2}+1}, \frac{|u|^{2}-1}{|u|^{2}+1}\right) .
$$

In terms of $u$, the energy densities $\epsilon_{2}$ and $\epsilon_{4}$ are given by

$$
\begin{gathered}
\epsilon_{2}=\frac{4}{\left(1+|u|^{2}\right)^{2}}\left(\nabla u \cdot \nabla u^{*}\right), \\
\epsilon_{4}=-8 \frac{\left(\boldsymbol{\nabla} u \times \nabla u^{*}\right)^{2}}{\left(1+|u|^{2}\right)^{4}} .
\end{gathered}
$$

The field equation can be rather simply expressed by $\boldsymbol{q}$ defined by

$$
\boldsymbol{q}=X \nabla u,
$$

with

$$
u=R \mathrm{e}^{i \Phi}, \quad R=|u|
$$

and

$$
X=2 \sqrt{\frac{c_{4}}{c_{2}}} \frac{1}{1+R^{2}}=\frac{1}{1+R^{2}},
$$


where $2 \sqrt{c_{4} / c_{2}}$ of the dimension of length has been set equal to 1 and $\boldsymbol{q}$ is dimensionless. The static field equation can be written as 7

$$
\nabla \cdot \boldsymbol{\alpha}+i \boldsymbol{\beta} \cdot \boldsymbol{\alpha}=0
$$

where $\boldsymbol{\alpha}$ and $\boldsymbol{\beta}$ are a complex and a real 3-vectors given by

$$
\begin{aligned}
& \boldsymbol{\alpha}=\boldsymbol{q}^{\star}-\boldsymbol{q}^{\star} \times\left(\boldsymbol{q} \times \boldsymbol{q}^{\star}\right), \\
& \boldsymbol{\beta}=\frac{1}{i}\left(u^{\star} \boldsymbol{q}-u \boldsymbol{q}^{\star}\right)=B \nabla \Phi, B=\frac{2 R^{2}}{1+R^{2}},
\end{aligned}
$$

respectively.

In this paper, we discuss some simple analytic solutions of the above equation. We shall consider mainly the vortex solutions of the form $R=R(\rho), \Phi=$ $m \phi$ with $(\rho, z, \phi)$ and $m$ being the cylindrical coordinates and an integer, respectively. We adopt an approximate analytic solution containing two adjustable parameters. Its form is fixed by the compatibility with the boundary condition and the singularity-structure necessitated by the field equation. The parameters are fixed so that the energy per unit length of vortex, $A_{m}$, becomes minimal. We find that the minimum of $A_{m}$ is proportional to $m$ approximately.

This paper is organized as follows. In Sec.2, we discuss the $m=0$ case briefly. In Sec.3, the above-mentioned approximate analytic solution for the $m \neq 0$ case is discussed. Sec.4 is devoted to summary.

\section{Some solutions in special cases}

We first consider the solutions of the form

$$
R=R(\rho, z), \quad \Phi=\Phi(\phi),
$$

where $(\rho, z, \phi)$ are cylindrical coordinates. Then we have

$$
\begin{aligned}
\boldsymbol{q} & =X \mathrm{e}^{i \Phi}\left(R_{\rho} \boldsymbol{e}_{\rho}+R_{z} \boldsymbol{e}_{z}+\frac{i R \Phi^{\prime}}{\rho} \boldsymbol{e}_{\phi}\right), \\
\boldsymbol{\alpha} & =\mathrm{e}^{-i \Phi}\left(C \boldsymbol{e}_{\rho}+D \boldsymbol{e}_{z}+F \boldsymbol{e}_{\phi}\right), \\
\boldsymbol{\beta} & =\frac{B \Phi^{\prime}}{\rho} \boldsymbol{e}_{\phi}, \\
C & =\left(X+\frac{R^{2} Y\left(\Phi^{\prime}\right)^{2}}{\rho^{2}}\right) R_{\rho}, \\
D & =\left(X+\frac{R^{2} Y\left(\Phi^{\prime}\right)^{2}}{\rho^{2}}\right) R_{z}, \\
F & =-i \frac{R \Phi^{\prime}}{\rho}\left[X+Y\left(R_{\rho}^{2}+R_{z}^{2}\right)\right], \\
Y & =2 X^{3}, \quad R_{z}=\frac{\partial R(\rho, z)}{\partial z}, \\
\Phi^{\prime} & =\frac{d \Phi(\phi)}{d \phi}, \quad R_{\rho}=\frac{\partial R(\rho, z)}{\partial \rho}, \quad R_{z},
\end{aligned}
$$


where $\boldsymbol{e}_{\rho}, \boldsymbol{e}_{z}$, and $\boldsymbol{e}_{\phi}$ are orthonormal unit vectors satisfying $\boldsymbol{e}_{\rho} \times \boldsymbol{e}_{\phi}=\boldsymbol{e}_{z}$, etc. We obtain $F_{\phi}=0$ from $\operatorname{Im}\{\nabla \cdot \boldsymbol{\alpha}+i \boldsymbol{\beta} \cdot \boldsymbol{\alpha}\}=0$. Then, taking the single-valuedness of $u$ into account, we are led to

$$
\Phi=m \phi, \quad m: \text { integer }
$$

On the other hand, from $\operatorname{Re}\{\nabla \cdot \boldsymbol{\alpha}+i \boldsymbol{\beta} \cdot \boldsymbol{\alpha}\}=0$ and $\Phi^{\prime}=m$, we have

$$
\begin{aligned}
& \nabla \cdot(X G \nabla R)+(G-1) \frac{R^{2}-1}{2 R}\left[1+2 X^{2}(\nabla R)^{2}\right]=0, \\
& G=1+\frac{2 m^{2} R^{2} X^{2}}{\rho^{2}} .
\end{aligned}
$$

In the $m=0$ case, it becomes $\triangle[\arctan R]=0$, whose solution is given by

$$
\begin{aligned}
\arctan R & =\sum_{n=0}^{\infty}\left(A_{n} r^{n}+\frac{B_{n}}{r^{n+1}}\right) \mathrm{P}_{n}(\cos \theta), \\
r & =\sqrt{\rho^{2}+z^{2}}, \quad \cos \theta=\frac{z}{r}
\end{aligned}
$$

where $\left\{A_{n}, B_{n}: n=0,1,2, \cdots\right\}$ are constants and $\mathrm{P}_{n}$ denotes the $n$-th Legendre polynomial. It should be noted that

$$
\arctan R=a_{1} \ln \rho+a_{2} \quad\left(a_{1}, a_{2}: \text { const. }\right)
$$

is a solution, too. These results indicate how Cho's solution 8$] u=\frac{\text { const. should }}{r}$ be generalized. They are, however, trivial in the sense that the interference effects between the $l_{2}(x)$ and $l_{4}(x)$ in the Lagrangian disappear in these solutions. For $m \neq 0$, we see that the simpler cases such as $R=f_{1}(z), R=f_{2}(r), R=$ $f_{3}(\theta), R=f_{4}(\eta), R=f_{5}(\xi)$ are not allowed since $G$ in Eq. (31) contains $\rho$, where $(r, \theta, \phi)$ and $(\eta, \xi, \phi)$ are polar- and toroidal- coordinates, respectively. Only the vortex solution $R=R(\rho)$, which is a solution of the 2-dimensional Faddeev model, is allowed.

\section{Approximate vortex solutions}

We hereafter consider the case $R=R(\rho)$ and $m \neq 0$. If we set

$$
R=\tan \frac{\xi}{2}, \quad \sigma=\frac{\rho}{m}
$$

we obtain

$$
\left(\sigma^{2}+2 \sin ^{2} \xi\right) \frac{d^{2} \xi}{d \sigma^{2}}+\sin 2 \xi\left(\frac{d \xi}{d \sigma}\right)^{2}+\left(\sigma-\frac{2}{\sigma} \sin ^{2} \xi\right) \frac{d \xi}{d \sigma}-2 m^{2} \sin 2 \xi=0
$$

Through a further change of variables

$$
V(\zeta)=-\tan ^{2} \xi=-\left(\frac{2 R}{R^{2}-1}\right)^{2}, \quad \zeta=\frac{\sigma^{2}}{\sigma^{2}+2}
$$


we obtain an algebraic differential equation

$$
\begin{aligned}
\frac{d^{2} V}{d \zeta^{2}}-\frac{1}{2}\left(\frac{1}{V}\right. & \left.+\frac{1}{\zeta-V}+\frac{3}{V-1}\right)\left(\frac{d V}{d \zeta}\right)^{2} \\
& +\left(\frac{1}{\zeta-1}+\frac{1}{\zeta-V}\right) \frac{d V}{d \zeta}-\frac{2 m^{2} V(V-1)}{\zeta(1-\zeta)^{2}(V-\zeta)}=0
\end{aligned}
$$

The solution of this equation can be explored in the following way. For a given $\zeta_{0}$ which is different from 0 and 1 , we assume that the solution $V(\zeta)$ near $\zeta=\zeta_{0}$ is given as

$$
V(\zeta)=\sum_{n=0}^{\infty} V_{n}\left(\zeta-\zeta_{0}\right)^{\alpha+n}, \quad 0<\zeta_{0}<1, \quad \alpha<0 .
$$

Then we are led to

$$
\alpha=-2
$$

and

$$
\begin{aligned}
V_{1} & =\frac{V_{0}}{\zeta_{0}-1}, \\
V_{2} & =\frac{\left(4 m^{2}+\zeta_{0}\right) V_{0}-4 \zeta_{0}\left(\zeta_{0}-1\right)^{2}\left(\zeta_{0}-3\right)}{12 \zeta_{0}\left(\zeta_{0}-1\right)^{2}}, \\
V_{3} & =-\frac{m^{2} V_{0}+2 \zeta_{0}^{2}\left(\zeta_{0}-1\right)^{2}}{6 \zeta_{0}^{2}\left(\zeta_{0}-1\right)^{2}},
\end{aligned}
$$

and so on. It turns out that $V_{0}$ and $\zeta_{0}$ are arbitrary and $V_{1}, V_{2}, V_{3}, \cdots$ are fixed by them.

Although $V_{n}$ can be determined order by order, it is difficult to conclude that the series (3.5) converges in some domain of $\zeta$ around $\zeta_{0}$. To obtain the radius of convergence of the series (3.5), if any, we must calculate $V_{n}$ for very large $n$, whose mathematical expression becomes quite complicated as $n$ gets large. The differential equation (3.4) suggests that the behavior of $V(\zeta)$ at $\zeta=0$ and $1(\rho=0$ and $\infty)$ may be different from that at ordinary points $0<\zeta<1$ $(0<\rho<\infty)$. It is also difficult to maintain the properties of $V(\zeta)$ near $\zeta=0$ and 1 by an infinite series of the form (3.5). We therefore consider an approximate $V(\zeta)$ which realizes the properties required by the lowest order analysis of $(3.4)$ around $\zeta=\zeta_{0}(\neq 0,1), \zeta=0$ and $\zeta=1$.

As was explained above, the behavior of $V(\zeta)$ in the neighborhood of $\zeta_{0}$ is given by $V(\zeta) \sim$ const. $\left(\zeta-\zeta_{0}\right)^{-2}$. We next consider the behavior of $V(\zeta)$ near $\zeta=0$. It turns out that the behavior $V(\zeta) \sim$ const. $\zeta^{\lambda}$ is compatible with (3.4) only when $\lambda=2 m$ or $\lambda=-2 m$. We here recall that, with the assumption (2.10), only $R=0$ and $R=\infty$ yield the vanishing energy density irrespective of the value of $\phi$. These values of $R$ imply $V(0)=0$, leading to $\lambda=2|m|$. In other words, if we require that the energy density is vanishing at $\rho=0$, the differential equation (3.4) and the assumption (2.10) lead us to

$$
V(\zeta) \sim \text { const. } \zeta^{2|\mathrm{~m}|}, \quad \zeta \sim 0
$$


Similarly, from the requirement that the energy density is vanishing at $\rho=\infty$ , we obtain

$$
V(\zeta) \sim \operatorname{const.}(\zeta-1)^{2|\mathrm{~m}|}, \quad \zeta \sim 1 .
$$

It is straightforward to see that the conditions $V(0)=V(1)=0$ correspond to the configurations $\boldsymbol{n}=(0,0,1)$ or $\boldsymbol{n}=(0,0,-1)$ at $\rho=0$ and $\infty$. Therefore we are here considering the configurations interpolating these configurations. In the example considered below (Fig.4), we obtain $V(\zeta)$ connecting $\boldsymbol{n}=(0,0,1)$ at $\rho=0$ and $\boldsymbol{n}=(0,0,-1)$ at $\rho=\infty$.

We note that, in the analysis of the hedgehog Skyrmion, the solution of the differential equation

$$
\begin{aligned}
\frac{d^{2} W}{d \eta^{2}}-\frac{1}{2}\left(\frac{1}{W}+\frac{1}{\eta-W}+\frac{3}{W-1}\right)\left(\frac{d W}{d \eta}\right)^{2} \\
\quad+\left[\frac{1}{2}\left(\frac{1}{\eta-1}+\frac{1}{\eta}\right)+\frac{1}{\eta-W}\right] \frac{d W}{d \eta}-\frac{W[(\eta+1) W-2 \eta]}{2 \eta^{2}(\eta-1)^{2}(W-\eta)}=0
\end{aligned}
$$

was investigated $[9]$ by a trial function

$$
W=\frac{-q \eta(\eta-1)^{2}}{\eta-p}, \quad q>0, \quad 1>p>0,
$$

which yielded a rather good value of the energy.

In the following, we consider the approximate solutions $V_{m}(\zeta)$ given by

$$
V_{m}(\zeta)=-q_{m} \frac{[\zeta(1-\zeta)]^{2 m}}{\left(\zeta-p_{m}\right)^{2}}, \quad m>0, \quad q_{m}>0, \quad 1>p_{m}>0
$$

which is compatible with the singularity-structure indicated by the differential equation and the boundary condition that the energy density vanishes at $\rho=$ $0, \infty$.

The energy $E_{F}$ corresponding to the assumption (2.10), which we denote by $E_{m}$, is now given by

$$
\begin{aligned}
E_{m} & =8 \pi c_{2} \int_{-\infty}^{\infty} A_{m} d z, \\
A_{m} & =\int_{0}^{1} W_{m} d \zeta \\
W_{m} & =\frac{m^{2}}{8} \frac{q_{m}[\zeta(1-\zeta)]^{2 m-1}}{\left(\zeta-p_{m}\right)^{2}+q_{m}[\zeta(1-\zeta)]^{2 m}} \\
& +\frac{1}{2} \frac{q_{m}[\zeta(1-\zeta)]^{2 m-1}\left[\zeta(1-\zeta)+m(2 \zeta-1)\left(\zeta-p_{m}\right)\right]^{2}}{\left\{\left(\zeta-p_{m}\right)^{2}+q_{m}[\zeta(1-\zeta)]^{2 m}\right\}^{2}} \\
& +\frac{1}{8} \frac{q_{m}^{2} \zeta^{4 m-2}(1-\zeta)^{4 m}\left[\zeta(1-\zeta)+m(2 \zeta-1)\left(\zeta-p_{m}\right)\right]^{2}}{\left\{\left(\zeta-p_{m}\right)^{2}+q_{m}[\zeta(1-\zeta)]^{2 m}\right\}^{3}} .
\end{aligned}
$$


We fix $p_{m}$ and $q_{m}$ so that they minimize $A_{m}$. For $m=1 \sim 4$, they are given as

$$
\left\{\begin{array}{lll}
m=1: & p_{1}=0.83, & q_{1}=4.0, \quad A_{1}=1.14 \\
m=2: & p_{2}=0.61, & q_{2}=2.3 \times 10, \quad A_{2}=2.23 \\
m=3: & p_{3}=0.56, & q_{3}=1.57 \times 10^{2}, \quad A_{3}=3.28 \\
m=4: & p_{4}=0.52, & q_{4}=1.36 \times 10^{3}, \quad A_{4}=4.34 .
\end{array}\right.
$$

It seems that $p_{m}$ approaches to 0.5 when $m$ becomes large. For the convenience of numerical estimation, we fix $p_{m}$ as 0.5 for $m$ larger than 4 and determine $q_{m}$ so as to minimize $A_{m}$. The result is given as follows:

$$
\left\{\begin{array}{lll}
m=5: & q_{5}=1.35 \times 10^{4} & A_{5}=5.42 \\
m=6: & q_{6}=1.45 \times 10^{5} & A_{6}=6.49 \\
m=7: & q_{7}=1.66 \times 10^{6} & A_{7}=7.57 \\
m=8: & q_{8}=1.96 \times 10^{7} & A_{8}=8.65 \\
m=9: & q_{9}=2.41 \times 10^{8} & A_{9}=9.74 \\
m=10: & q_{10}=3.06 \times 10^{9} & A_{10}=10.8 .
\end{array}\right.
$$

The results for $A_{m}$ and $q_{m+1} / q_{m}$ are given in Fig.1 and Fig.2, respectively. We are then led to the approximate formulae

$$
A_{m}=a+b m, \quad a=0.052, b=1.076
$$

and

$$
\frac{q_{m+1}}{q_{m}}=c\left(1-d \mathrm{e}^{-f m}\right), \quad c=14.0, d=0.769, f=0.234 .
$$

Since the infinite product $\prod_{m=0}^{\infty}\left(1-d \mathrm{e}^{-f m}\right) \equiv B$ converges to 0.00592 , we have $\log q_{m} \sim m \log c+\log B$ for large $m$. From the fact that $a \sim 0$ and $b \sim A_{1}$, it looks like that there exists an atom-like object $\mathrm{Q}$ with energy $b$ and unit $m$ and that the assembly of $m$ Q's constitutes the configuration with $m$.

With the original variables, the energy density $K(\rho)$ defined by $A_{1}=\int_{0}^{\infty} K(\rho) d \rho$ and $R(\rho)$ for $m=1$ are given in Fig.3 and Fig.4, respectively.

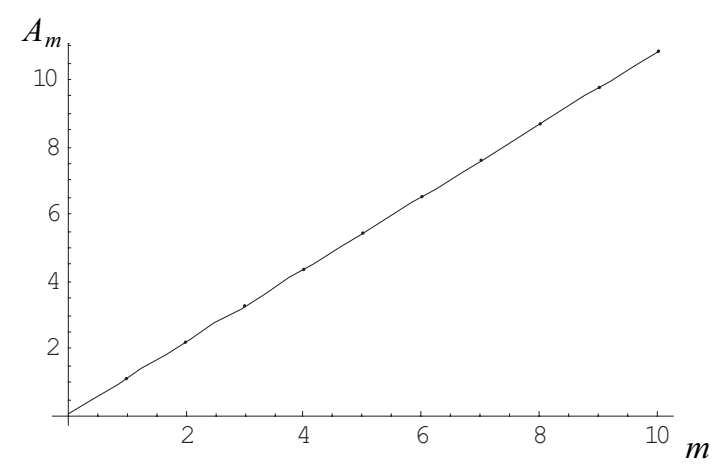

Figure 1: $A_{m}$ vs. $m$ 


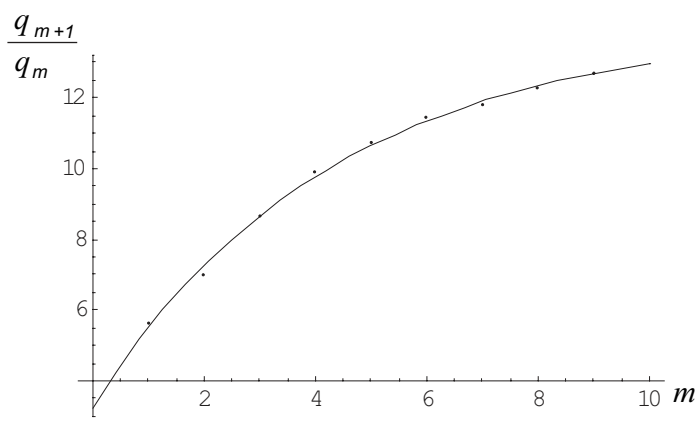

Figure 2: $\frac{q_{m+1}}{q_{m}}$ vs. $m$

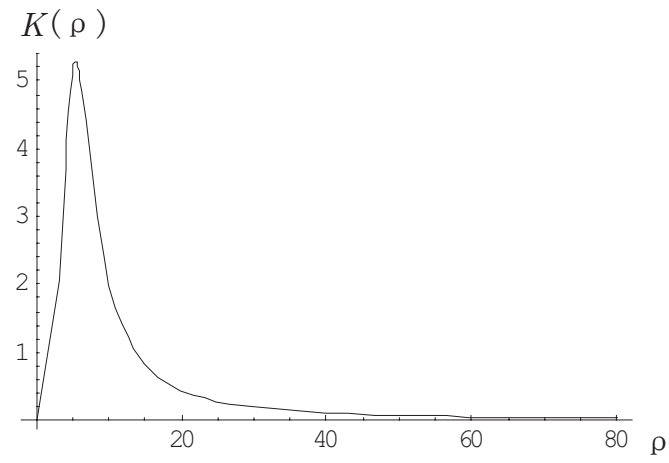

Figure 3: Energy density $K(\rho)$ vs. $\rho$

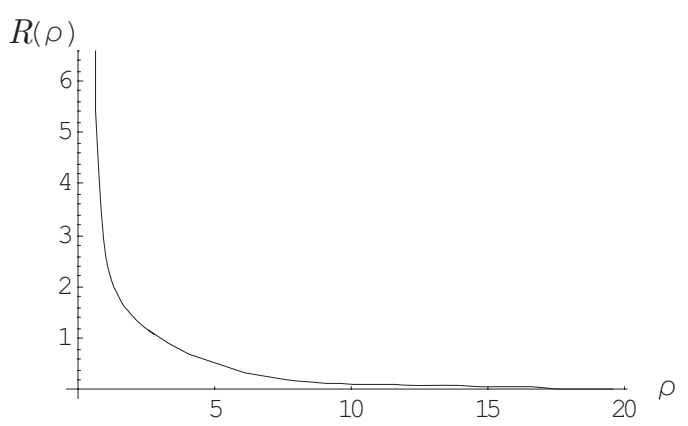

Figure 4: $R(\rho)$ vs. $\rho$ 


\section{Summary}

We have examined the vortex solutions of the Faddeev model. Through the change of variables, the field equation was converted to an algebraic differential equation containing an integer-parameter $m$. Its approximate solution was parameterized with the aid of two parameters $p_{m}$ and $q_{m}$. They were fixed so that the energy per unit length of vortex, $A_{m}$, became minimal. It was observed that the minimum of $A_{m}$ is proportional to $m$ approximately. The numerical analysis of the Faddeev model made so far clarified the knot structure of the genuine three-dimensional solitons. We hope that our two-dimensional analysis might be helpful for the understanding of the latter since knot-solitons may be regarded as the bended and twisted vortices.

\section{Acknowledgments}

This research was partially supported by the National Natural Science Foundation of China (Grant No. 10601031).

\section{References}

[1] L. Faddeev, Lett. Math. Phys. 1, (1976) 289.

[2] L. Faddeev and A. J. Niemi, Phys. Rev. Lett. 82, (1999) 1624.

[3] T.H.R. Skyrme, Nucl. Phys.31 (1961) 556.

[4] R. A. Battye and P. M. Sutcliffe, Phys. Rev. Lett. 81, (1998) 4798.

[5] R. A. Battye and P. M. Sutcliffe, Phys. Rev. Lett. 79, (1997) 363.

[6] J. Hietarinta and P. Salo, Phys. Lett. B 451, (1999) 60.

[7] M. Hirayama and C.-G. Shi, Phys. Lett. B652,(2007) 384.

[8] Y. M. Cho, Phys. Rev. Lett. 87, 252001 (2001).

[9] J. Yamashita and M. Hirayama, Phys. Lett. B642,(2006) 160. 